\title{
Linx
}

Revue des linguistes de l'université Paris X Nanterre

49 | 2003

L'actualité des notions d'interlangue et d'interaction exolingue

\section{Réflexion autour du concept d'interlangue pour décrire des variétés non natives avancées en français}

\section{Stéphanie Galligani}

\section{OpenEdition}

Journals

Édition électronique

URL : http://journals.openedition.org/linx/562

DOI : $10.4000 /$ linx.562

ISSN : 2118-9692

\section{Éditeur}

Presses universitaires de Paris Nanterre

Édition imprimée

Date de publication : 1 décembre 2003

Pagination : 141-152

ISSN : 0246-8743

\section{Référence électronique}

Stéphanie Galligani, «Réflexion autour du concept d'interlangue pour décrire des variétés non natives avancées en français », Linx [En ligne], 49 | 2003, mis en ligne le 18 mars 2011, consulté le 30 avril 2019. URL : http://journals.openedition.org/linx/562; DOI : 10.4000/linx.562 


\title{
Réflexion autour du concept d'interlangue pour décrire des variétés non natives avancées en français
}

\author{
Stéphanie Galligani \\ Université Paris III - Sorbonne Nouvelle \\ et LIDILEM - Grenoble
}

\section{Introduction}

Depuis plus d'une vingtaine d'années, de nombreuses recherches empiriques sur l'acquisition spontanée d'une langue étrangère par des adultes immigrés en milieu social ont vu le jour ; beaucoup d'entre elles ont porté sur la description des processus d'acquisition et sur l'évolution des connaissances. On peut citer, entre autres, les recherches coordonnées par la Fondation Européenne de la Science (ESF) ou encore les travaux des deux équipes du GRAL ${ }^{1}$ (GRAL Paris et GRAL Université de Provence) dont l'objectif a été d'étudier la compétence linguistique et communicative en langue du pays d'accueil chez des migrants adultes monolingues, peu scolarisés et ne recevant que peu de cours de français. S’il est évident que ces divers travaux ont permis de mettre en lumière les processus d'acquisition de la langue d'accueil (désormais langue d'accueil $=$ LA) en accordant une attention particulière à la mise en place progressive des hypothèses de construction de l'interlangue, il n'en est pas moins vrai qu'aujourd'hui très peu d'observations ont été entreprises sur les pratiques effectives en français parlé chez ces migrants de longue date. Il est d'autant plus surprenant que ce domaine de recherche ait été écarté des préoccupations des chercheurs dans la mesure où celui-ci pourrait s'inscrire dans le prolongement des premiers travaux du programme ESF sur le volet acquisitionnel.

C'est dans cette perspective de recherche qu'ont été réalisés mes travaux en doctorat $^{2}$ sur les pratiques langagières en français parlé par des migrants espagnols de longue date à partir d'entretiens individuels réalisés auprès de quatre sujets installés en France depuis plus d'une vingtaine d'années. Cette recherche m'a permis, entre autres, d'approfondir les divers travaux réalisés sur l'acquisition d'une langue étrangère en

\footnotetext{
${ }^{1}$ Groupe de Recherche sur l'Acquisition des Langues.
}

${ }^{2}$ Voir Costa Galligani 1998 (a). 
Stéphanie Galligani

contexte social (même si leur intérêt se limite à la description des compétences linguistiques d'apprenants en LA lors des premiers stades du processus d'acquisition) mais surtout d'évaluer les différents outils utilisés pour décrire les comportements langagiers d'apprenants en langue étrangère. Un des outils privilégiés dans la mise au jour de leur répertoire langagier en LA est celui d'interlangue, concept nodal en psycholinguistique de l'acquisition. Mon objectif a été de déterminer la pertinence de ce concept dès lors qu'il s'agit de caractériser des variétés non natives avancées de français chez des migrants.

Cet article se limitera d'abord à rappeler en détail les propriétés du concept d'interlangue qui, dans une perspective acquisitionnelle, sert à caractériser les systèmes intermédiaires par lesquels passe l'apprenant en langue étrangère. Il se poursuivra ensuite par une réflexion sur certaines limites d'application d'un tel concept pour décrire des situations de contacts de langues en contexte migratoire.

\section{Le concept d'interlangue : définition et propriétés}

Sans vouloir retracer l'évolution des différentes approches sur les processus acquisitionnels mis en œuvre par un apprenant en langue étrangère, il me semble pourtant nécessaire de rappeler que l'analyse des erreurs, qui a suscité de nombreuses interrogations et des ouvertures dans le domaine de l'apprentissage d'une langue étrangère, a permis que l'on s'intéresse à la langue de l'apprenant, c'est-à-dire à son interlangue définie comme «la connaissance et l'utilisation non-natives d'une langue quelconque par un sujet non-natif et non-équilingue, c'est-à-dire un système autre que celui de la langue cible mais qui, à quelque stade d'apprentissage qu'on l'appréhende, en comporte certaines composantes » (Besse et Porquier 1991 : 216). Dès lors, une discussion terminologique s'engage sur la notion d'interlangue au niveau de ses différentes définitions et acceptions dans les nombreux travaux déjà effectués dans ce domaine.

\section{Sa définition}

Corder part de l'idée que «quelqu'un qui apprend une langue étrangère (LE) possède une langue en ce sens que son activité langagière obéit à des règles, et que l'on peut par conséquent, en principe, la décrire en termes linguistiques » (Corder 1980 : 29). Ce que ce chercheur nomme «la langue de l'apprenant» a reçu diverses dénominations selon les auteurs. Ainsi figure dans le paragraphe du chapitre X de la Grammaire et didactique des langues, à la suite de Frauenfelder et al. (1980 : 44), un rappel des différentes terminologies complétées par le nom des auteurs et la date de parution: "Ce que l'on a dénommé, ici ou là, système approximatif (Nemser 1971), compétence transitoire (Corder 1967), dialecte idiosyncrasique (Corder 1971), système intermédiaire (Porquier 1974), interlangue (Selinker 1972), système approximatif de communication, langue de l'apprenant ou système approché (Noyau 1976) [...] recouvre, malgré certaines dispersions théoriques ou méthodologiques, un même objet [...]. C'est ce que nous avons précédemment appelé grammaire intériorisée par l'apprenant, et que nous appellerons également ici interlangue» (Besse et Porquier 1991: 216). 
Quelles que soient les terminologies qui opposent les diverses conceptions de l'interlangue, on retrouve partout l'idée d'une structuration progressive des connaissances d'apprenants en langue étrangère, d'une complexification par laquelle la compétence intermédiaire se rapproche de l'objectif fixé au départ par les apprenants, à savoir la langue cible.

Une des premières orientations de recherche sur l'interlangue a été conduite par Selinker, chercheur attaché aux aspects linguistiques et psycholinguistiques de l'apprentissage d'une langue étrangère par des adultes, avec l'élaboration du terme «interlanguage » (traduit par «interlangue» en français) pour rendre compte des connaissances intermédiaires des apprenants au cours de l'apprentissage d'une langue étrangère. Sa définition en termes psycholinguistiques postule l'existence de «a latent psychological structure within which interlingual identifications and the processes and strategies underlying second-language learning are located [...] the latent psychological structure is actived after puberty, whenever an individual attempts to express meaning in a second language $»^{3}$ (Selinker et al., $1975: 215$ ). Comme le rappellent Frauenfelder et al. (1980 : 45), l'élaboration de ce modèle implique un «âge critique» en matière d'apprentissage des langues et qu'au-delà de cette frontière, des contraintes biologiques viendraient altérer la capacité d'apprentissage linguistique. Autrement dit, un apprentissage « réussi » (successul learning) serait, selon Selinker, conditionné par la réactivation de la structure latente; en revanche, un apprentissage qui n'aboutirait pas à une maitrise semblable à celle d'un locuteur natif de cette langue signifierait que l'apprenant ne serait pas parvenu à activer cette structure latente(attempted learning). L'avancement des travaux en matière d'apprentissage d'une langue étrangère, élargi à l'acquisition par des apprenants plus jeunes en âge que ceux étudiés préalablement par Selinker, a finalement remis en cause l'existence d'un âge critique et les résultats ont mis en lumière les profondes imperfections de son modèle (voir Vogel 1995). En particulier, les travaux menés par Neufeld (1979), qui oppose «capacité linguistique » et "performance en langue étrangère», montrent que l'incapacité de l'adulte à apprendre une langue étrangère tient en partie à sa non-réussite dans la production. Dès lors, la définition de l'interlangue s'inscrit dans une hypothèse plus large :

The Interlanguage (IL) hypothesis claims that second-language speech rarely conforms to what one expects native speakers of the TL [target language] to produce, that it is not an exact translation of the NL [native language], that it differs from the TL in systematic ways, and that the forms of the utterances produced in the second language by a learner are not random. This IL hypothesis proposes that the relevant data of a theory of second language learning must be the speech forms which result from the attempted expression of meaning in a second language. [...] This important criterion is that the second-language speaker is attempting to express

\footnotetext{
3 «une structure psychologique latente au sein de laquelle se trouvent des identifications interlinguales, les processus et les stratégies sous-jacentes de l'apprentissage d'une seconde langue [...] la structure psychologique latente est activée après l'âge de puberté, chaque fois qu'un individu tente d'exprimer du sens en langue seconde » (notre traduction).
} 
Stéphanie Galligani

meaning as opposed to practising structured exercises in a classroom ${ }^{4}$ (Selinker et al., $1975: 140)$

La description de l'interlangue de sujets apprenant une langue étrangère, c'està-dire « la description de la grammaire sous-jacente à leurs comportements verbaux en langue seconde » (Py 1980 : 32), paraît comporter des traits spécifiques qui méritent d'être examinés.

\section{Ses propriétés}

Parmi l'ensemble des observables qui sous-tend l'hypothèse de l'interlangue, Selinker et al. distinguent: "First, the stability over time of certain errors and other surface forms in learner-language systems. Second, the mutual intelligibility that appears to exist among speakers of the same IL. Third, the phenomenon of backsliding or the regular reappearance in bilingual speech of fossilized errors that were thought to be eradicated. And fourth, the systematicity of the IL at one particular point in time $\aleph^{5}$ (1975: 141). A ces composantes viennent s'ajouter d'autres caractéristiques, non moins pertinentes que les précédentes, à savoir celles de dynamique (instabilité, variabilité), de système (systématicité) et de spécificité (perméabilité).

\section{a) L'instabilité}

L'intérêt théorique du champ de recherches sur l'apprentissage d'une langue étrangère a participé à l'introduction, dans la problématique de l'interlangue, de la notion de «microsystème », réflexion qui a été amorcée par Berthoud et Py (1979) à la suite de Gentilhomme. Le microsystème est défini par ces auteurs comme « un être vivant, c'est-à-dire partiellement contradictoire et déséquilibré, susceptible de transgresser ses propres lois de fonctionnement [...]. C'est la possession de tels traits qui donne au microsystème son dynamisme, c'est-à-dire les ressorts qui entraînent les modifications continues en son sein » (1979 : 23). Les microsystèmes se présentent alors comme des ensembles autonomes de telle sorte que chacun peut fonctionner selon des principes originaux et spécifiques et qui ne sont pas généralisables à l'ensemble du système (Py 1980). Ces définitions permettent ainsi de mettre en

\footnotetext{
4 «L'hypothèse de l'interlangue (IL) soutient que le discours en langue seconde se conforme rarement à ce que l'on attend comme production de locuteurs natifs de la LC [langue cible], que ce n'est pas une traduction exacte de la LM [langue maternelle], qu'il diffère de manière systématique de la LC, et que les formes produites en langue seconde ne sont pas arbitraires. Cette hypothèse de l'IL propose que les données pertinentes d'une théorie de l'apprentissage de la langue seconde doivent être les formes du discours qui résultent de la tentative d'exprimer du sens en langue seconde. Le critère important est que le locuteur de la langue seconde tente d'exprimer du sens contrairement à la pratique des exercices structuraux en classe » (notre traduction).

5 « Premièrement, la stabilité dans le temps de certaines erreurs et d'autres formes de surface dans les systèmes linguistiques des apprenants. Deuxièmement, l'intercompréhension qui semble exister parmi les locuteurs d'une même IL. Troisièmement, le phénomène de régression ou la réapparition régulière, dans le discours bilingue, d'erreurs fossilisés qu'on croyait disparues. Et quatrièmement, la systématicité de l'IL à un certain moment donné » (notre traduction).
} 
évidence l'instabilité de l'interlangue qui dépend directement de l'existence de contradictions internes. Ce qui conduit Py à introduire la notion d'« hétérogénéité », comme élément constitutif de l'interlangue, pour rendre compte des modalités de construction, de réorganisation et de relation des microsystèmes entre eux, mais aussi de la manière dont l'analyste les appréhende. Le travail du chercheur consiste donc à décrire ces microsystèmes, non pas en recensant séparément les formes correctes et incorrectes mais «à tenter de reconstituer les règles sous-jacentes à l'ensemble» (Besse \& Porquier 1991 : 220). Ces règles et la variabilité dans leur application ne renvoient pas à des règles correspondantes de la langue maternelle, ni même à celles de la langue cible, mais à une organisation spécifique. Il en résulte qu'une interlangue est bien un système linguistique autonome qui doit être décrit comme tel, mais un système essentiellement instable qui est soumis «à des forces (internes et externes) qui exigent de l'analyste un souplesse continuelle» (Py 1980 : 32). Ainsi, son étude doit tenir compte de son caractère instable qui est une des autres composantes essentielles de l'interlangue.

\section{b) La perméabilité}

Un autre aspect qui relève des propriétés linguistiques de l'interlangue est sa perméabilité. Celle-ci paraît être, dans le modèle de Adjemian (1976), un des traits caractéristiques distinguant les interlangues des langues naturelles. C'est dans cette optique qu'il analyse le phénomène d'interlangue comme suit :

dans une situation où l'apprenant tente de communiquer en langue-cible (c'est-à-dire au moyen de son interlangue), il aura tendance à simplifier, à schématiser les aspects de sa grammaire en évolution qui provoquent le plus de difficultés, qui bloquent le plus la communication. C’est ici [...] que la perméabilité de son IL laissera violer sa systématicité interne, en acceptant des surgénéralisations, des simplifications, ou d'autres modifications d'une fonction linguistique quelconque qui lui est propre (1976: 09).

Ces modifications (surgénéralisation et simplification) ${ }^{6}$, qui sont interprétables comme des stratégies d'apprentissage, montrent que l'apprenant, pour répondre à des exigences communicatives, peut recourir à des moyens qui sont des manifestations du caractère perméable de son interlangue. Mais pour Frauenfelder et al. (1980 : 49), la perméabilité n'est pas un trait spécifique à l'interlangue. L’un des arguments qui les

\footnotetext{
${ }^{6}$ On entend par «surgénéralisation» ou «transfert intralingual»: «l'application abusive, à un domaine ou un élément de la langue, d'une règle élaborée dans un autre domaine où elle est pertinente» (Bautier-Castaing 1980:96). La séquence «il a couré» rend compte d'une surgénéralisation où la forme du participe passé est basée sur la conjugaison générale (exemple emprunté à Selinker et al. 1975 : 148). La stratégie de «simplification» correspond, elle, à : «la suppression (en référence au système de la langue cible ([LC]) par l'apprenant des désinences verbales, nominales, des redondances, des marques liées aux contraintes de cooccurrence, le recours à l'intonation, à la parataxe » (Bautier-Castaing 1980 : 96). Cette stratégie s'illustre dans la séquence suivante où l'infinitif est utilisé pour un présent: " mon maman et mon papa aller à Glendon» (exemple emprunté à Selinker et al., 1975 : 149).
} 
conduit à renoncer à cette spécificité tient au fait que la perméabilité, en tant que «manifestations d'hypothèses concurrentes sur l'organisation d'une partie de la grammaire de la LC, que l'apprenant est en train de vérifier», est caractéristique de tout locuteur bilingue dans la mesure où elle est liée au phénomène d'apprentissage.

Pour Py, le caractère évolutif de l'interlangue renvoie à la perméabilité de ses microsystèmes définis par des «frontières originales, différentes la plupart du temps de celles que se donnerait la linguistique contrastive» (1980 : 47). Au fil de l'apprentissage, ces «frontières originales » tendent à évoluer par l'apport de données nouvelles qui vont venir modifier, pour qu'elles puissent être intégrées, la structuration des microsystèmes. Ces microsystèmes apparaissent non pas comme des entités homogènes et cohérentes «mais comme des ensembles incomplets et déséquilibrés, voués même à la disparition, si la structure n'arrive pas à se former et à se consolider » (Rattunde 1980 : 47).

\section{c) La variabilité/la systématicité}

La variabilité qui reflète un état transitoire et donc par surcroit le caractère dynamique de l'interlangue, s'observe à plusieurs niveaux. Tout d'abord, elle est étroitement liée à la diachronie, c'est-à-dire au découpage de l'acquisition en étapes. Noyau (1976 : 54) suggère qu'il ne s'agit pas à proprement parler d'étapes mais plutôt d' " états » dans le processus d'acquisition d'une langue étrangère, sorte de «coupes synchroniques» qui rendent compte à chaque moment de l'apprentissage d'une cohérence interne. Pour Berthoud et Py (1979), ce découpage est arbitraire du fait qu'il ne correspond pas systématiquement à des paliers significatifs dans l'apprentissage. Et c'est souvent la raison pour laquelle certains travaux, en raison d'une approche contrastive, vont comparer des états successifs de l'apprentissage.

On peut également décrire la variabilité de l'interlangue d'un apprenant au regard des productions qui renferment des formes correctes correspondant aux formes et aux règles de la langue cible et des formes incorrectes. Mais cette variabilité que tente de dégager le linguiste ne peut se réduire à l'opposition énoncés corrects/énoncés incorrects par rapport aux règles de la langue cible car chaque production est à analyser comme le résultat d'une succession d'hypothèses hiérarchisées, de telle façon que la source d'une malformation peut être localisée dans la chaîne des opérations de prédiction. Il revient donc au chercheur de situer l'origine grammaticale de la malformation et de la compléter par une explication des énoncés observables. Autrement dit, l'examen du comportement langagier d'un apprenant doit considérer d'une part, la grammaire par laquelle il parvient à organiser les unités en phrases et d'autre part, les stratégies d'apprentissages (par exemple, la surgénéralisation) qui se manifestent par «une chaine d'explications causales » (Py 1980 : 35). De plus, la variabilité de l'interlangue s'observe par rapport aux situations de production linguistique. Les facteurs susceptibles d'influencer sensiblement le comportement langagier de l'apprenant sont à chercher du côté des situations et des procédures de sollicitation des données, appelées encore tâches. En effet, pour Frauenfelder et Porquier, les tâches qui consistent en principe en une activité langagière sont un moyen efficace pour "décrire l'état de l'interlangue à un stade donné ou à plusieurs stades successifs, de comprendre le processus d'apprentissage, 
de vérifier des hypothèses, sans que les résultats s'appliquent a priori à l'enseignement » (1980: 62).

Si l'on peut définir une interlangue par sa variabilité, on peut paradoxalement la caractériser par sa systématicité, terme qui sert à désigner la cohérence interne d'une interlangue à un moment donné. Contrairement à celle des langues naturelles, la systématicité d'une interlangue peut être assez complexe et comporter des variations. En effet, pour servis des facteurs situationnels et/ou communicationnels, l'apprenant va remettre en cause la systématicité par l'intériorisation de règles nouvelles perturbant ainsi l'assemblage de sa grammaire intériorisée, ou alors par la modification (sous forme de surgénéralisation ou de distorsion) d'une règle déjà existante. Dans cette perspective, la systématicité devient la résultante d'un parcours d'apprentissage qui l'a construite et qui peut la transformer plus ou moins rapidement, « et non la somme de savoirs successifs additionnés, comme un mur qui se construit de briques » (Besse et Porquier 1991 : 227). Nemser prend appui sur cette hypothèse lorsqu'il décrit le système approximatif de l'apprenant: "The speech of a learner, according to the assumption, is structurally organized, manifesting the order and cohesiveness of a system, although one frequently changing with atypical rapidity and subject to radical reorganization through the massive intrusion of new elements as learning proceeds ${ }^{7}$ (1971 : 116). Ainsi, le caractère évolutif de l'interlangue se définit par son instabilité et sa variabilité. Et ces traits sont centraux dans une théorie de l'interlangue dans la mesure où l'apprenant passe par des états successifs, chacun d'entre eux se construisant sur un état antérieur en le modifiant.

\section{d) La simplification/la complexification}

Si l'on compare des interlangues de sujets apprenant une langue étrangère à la langue cible, on est tenté d'y voir des stratégies de simplification ${ }^{8}$, semblables à celles que l'on pourrait rencontrer dans les pidgins. L'idée de simplification a été développée par Nemser qui reconnaît dans les interlangues d'apprenants des « utility systems, such as specialized 'little' languages of limited semantic function $» 9$ (1971 : 118). Autrement dit, la langue des apprenants reflèterait des systèmes de règles simplifiés par rapport à la langue à apprendre. Comme le font remarquer Besse et Porquier (1991 : 222), cette idée tient en partie au fait que la morphologie est plus pauvre (paradigmes verbaux restreints, peu de formes pour le genre et le nombre, etc.). Mais il existe un réel danger d'y voir un système simplifié - sorte de grammaire réduite - car cela pourrait conduire

\footnotetext{
${ }^{7}$ «Le discours d'un apprenant, selon l'hypothèse, est structurellement organisé et manifeste l'ordre et la cohésion d'un système, bien que celui-ci change fréquemment avec une rapidité atypique et est soumis à une réorganisation radicale par le biais d'une intrusion massive d'éléments nouveaux au fur et à mesure que l'apprentissage avance » (notre traduction).

${ }^{8}$ Le terme de «simplification » est très ambigu dans la mesure où on l'emploie indistinctement pour rendre compte des productions observées qui renvoient aux caractéristiques intrinsèques de l'interlangue (résultats), et aux processus d'apprentissage, c'est-à-dire les opérations sous-jacentes à la sélection des formes pour construire son interlangue (processus) (voir Porquier 1977 : 32).

${ }^{9}$ «systèmes utilitaires tels que des petites langues d'une fonction sémantique limitée » (notre traduction).
} 
le linguiste à ignorer sa complexité interne. S'il est certain qu'une interlangue présente un système moins développé et moins complexe que celui de la langue cible, en revanche ce qui la caractérise «c'est sa complexification progressive - sauf fossilisation ou éventuelle régression - et le processus de complexification que reflètent la nature et l'évolution de ses règles internes » (Besse \& Porquier 1991 : 223). Autrement dit, l'acquisition d'une langue étrangère est à concevoir comme un modèle dynamique montrant un processus de complexité croissante de l'interlangue.

En point de conclusion, on retiendra globalement que l'interlangue doit s'analyser comme un ensemble de microsystèmes autonomes de telle sorte que chacun peut fonctionner selon des règles originales et spécifiques qui évoluent par l'apport de données nouvelles et qui vont venir modifier, pour qu'elles puissent être intégrées, la structuration des microsystèmes. Ses propriétés intrinsèques reposent donc sur son instabilité, sa variabilité et sa perméabilité ; celles-ci permettant de la différencier d'une langue naturelle. Enfin, son système est à appréhender comme différent de celui de la langue maternelle et aussi de celui de la langue cible. C'est pourquoi il n'est pas concevable de la définir dans une perspective contrastive, ce qui en donnerait une visions fausse et réductrice.

\section{Des limites d'application du concept d'interlangue}

On a essayé jusqu'ici de dégager les différentes propriétés de l'interlangue. Se pose, à présent, le problème de savoir si ce concept et toutes ses propriétés restent un outil d'analyse pour caractériser des variétés non natives avancées de français chez des migrants espagnols de longue date.

Adopter une telle perspective reviendrait alors à considérer que les compétences linguistiques développées par les migrants en LA s’apparenteraient à des «systèmes approximatifs", à des «compétences transitoires", à des «systèmes intermédiaires » ou encore à des «systèmes approchés", selon les différentes terminologies évoquées précédemment. Arrêtons-nous un instant sur les incidences d'un tel choix conceptuel. Il est clair que son emploi impliquerait, dans le contexte concerné, le maintien des sujets dans un processus d'appropriation de la LA alors qu'eux-mêmes déclarent ne plus être engagés dans ce dernier (voir Costa Galligani 1998b).

Ex.1. mais le jour où je vu que les gens en face de moi comprenaient ce que je disais ben c'était arrêté + c'est idiot mais je n'avais à mon humble avis je n'avais aucun envie je n'avais aucun besoin de continuer alors alors évidemment c'est une bêtise mais pour moi non c'est bon [...] pou moi ça suffisait [...] je ne voyais pas le côté pratique je voulais pas faire une carrière de machin ni ni ni de lettre ni autre donc voilà pourquoi c'est tout (Juan, 1, 8, 10)

De plus, cela reviendrait à dire qu'ils ne sont jamais parvenus à atteindre cette fameuse cible que représente le français comme en témoignent les formes idiosyncrasiques et les marques transcodiques (sous forme de transferts interlinguaux) relevées dans leurs discours. 
Ex.2 + alors Nathalie il a il a fini son bac donc l'année prochaine il a fait un année sabbatique là et puis Béatrice qui a quatorze ans maintenant et qui travaille très très très bien + c'est $i l$ est brune presque aussi belle que toi $i l$ est magnifique aussi + (Juan 2, 12, 26)

[référent $1:$ Nathalie; référent $2:$ Béatrice repris par le pronom clitique i]

Ex.3 + j'avais j’avais convalidé mes études de la marine espagnole donc pour la marine marchande (Juan 2, 9, 11)

[création lexicale à partir du terme espagnol convalidar et du terme français valider]

Ex. 4 bon ben je pris je suis bilangue voilà + mais bilangue par nature hein c'est-à-dire pas + enfin por obligation por obligation (Pedro 2, 1, 17)

[création lexicale selon l'opération : langue + langue = bi-langue !]

D'un point de vue linguistique, le danger pour le chercheur serait alors d'analyser ces productions comme des «erreurs » ou des « déviances » par rapport à une norme monolingue de réalisation et non comme la trace manifeste d'opérations inter- ou intralinguales (voir Costa Galligani 1998). Par ailleurs, le but des sujets étudiés est moins d'atteindre un état de connaissance "parfait» en français que de pouvoir communiquer dans cette langue, quelle que soit la situation de communication. En effet, ce qui est visé par les sujets n'est pas la langue cible mais plutôt une compétence linguistique qu'ils jugent suffisante pour pouvoir se «débrouiller » dans cette langue. De ce fait, il apparait plus légitime d'envisager le processus par lequel ces sujets (le terme d'apprenant - au sens didactique du terme est d'emblée à écarter sinon on les inscrit d'emblée dans un processus d'acquisition) se sont appropriés la LA comme un processus plutôt achevé.

Mais peut-on encore parler d'interlangue pour décrire les connaissances linguistiques emmagasinées au terme d'un long processus d'appropriation achevé ? Cette question soulève un autre problème, celui d'une durée que recouvrirait le concept d'interlangue pour analyser des situations linguistiques complexes. A partir de quelle étape du processus d'acquisition ce concept, plus fréquemment utilisé pour décrire les premières étapes du processus, ne devient-il plus opératoire? Existe-t-il un stade critique (qui resterait cependant à définir!) au-delà duquel on pourrait imaginer qu'il deviendrait inopérant à caractériser les connaissances d'un locuteur dans une langue étrangère?

Enfin, un dernier argument qui m'amène à écarter définitivement la notion d'interlangue tient à l'étymologie de ce terme. Le préfixe «inter-», directement emprunté du latin «inter» (qui signifie « entre») et employé comme préfixe dans la composition de certains mots, marque au sens le plus général, la séparation, l'espacement et la réciprocité. Si l'on s'en tient à la définition du Trésor de La Langue Française (1983 : 405), « inter » renvoie à deux notions :

$1^{\circ}$ «Inter- désigne la partie de l'espace (ou du temps) séparée par deux éléments ou délimitée par plusieurs éléments ». Lorsque les mots construits sont des substantifs et que le deuxième élément est un substantif, ils désignent soit « un espace délimité 
Stéphanie Galligani

par deux éléments», soit "une période intermédiaire entre deux périodes caractérisées par l’activité spécifique » (ex. interphase, intersaison, etc.) ;

$2^{\circ}$ «Inter- exprime la notion de relation, de réciprocité ». Lorsque les mots construits sont des adjectifs, le deuxième élément est un adjectif de relation (ex. interbancaire) ou des substantifs d'action ou d'état (ex. interclassement).

C'est dans cette première définition que l'on peut reconnaître la formation $\mathrm{du}$ terme « interlanguage » de Selinker (1972) : «language » renvoyant à la notion de système linguistique (on ne reviendra pas sur les problèmes qu'a posé la traduction de ce terme générique en français), « inter-» pour marquer une période intermédiaire. L'association lexicale donne en français le terme interlangue pour désigner un stade intermédiaire dans l'appropriation d'une langue étrangère, située entre deux autres stades que représenteraient respectivement la langue source et la langue cible. Et c'est précisément cette façon d'envisager l'interlangue comme une formation linguistique originale "entre" deux langues qui me conduit finalement à son renoncement. Dans un phénomène de contacts de langue, il ne viendrait jamais à l'esprit du linguiste d'appréhender le système comme quelque chose situé entre deux langues, mais plutôt comme une configuration linguistique originale à composante bilingue.

L'ensemble des problèmes que l'on vient d'évoquer montre assez bien l'inadéquation de la notion d'interlangue pour décrire le comportement langagier des sujets étudiés. Il ne s'agit pas là d'affaiblir cet outil dont le bien-fondé présente des avantages difficilement contestables aujourd'hui mais d'envisager les limites de son application dès lors qu'il s'agit de caractériser des situations sociolinguistiques complexes. On parvient donc à la conclusion qu'une approche strictement acquisitionnelle ne peut à elle seule répondre à la spécificité du contexte étudié même si les productions langagières recueillies partagent certaines propriétés de l'interlangue, en particulier l'instabilité et la perméabilité des microsystèmes témoignant ainsi de la présence d'opérations inter et/ou intralinguales ou encore la variabilité dans les réalisations linguistiques à l'intérieur de ces microsystèmes (voir Costa Galligani 2001).

\section{Conclusion}

Pour définir le comportement langagier des sujets étudiés, on pourrait opter pour des concepts plus neutres et plus larges, comme par exemple le parler des sujets pour rendre compte d'un système linguistique particulier, les variétés du français parlé pour signaler des zones de turbulences linguistiques à l'intérieur des microsystèmes, ou encore les pratiques langagières pour la dimension discursive. Mais il serait sans doute plus juste de retenir une notion qui puisse rendre compte à elle seule de toutes ces composantes, à savoir linguistique, discursive et surtout variationniste. Dans l'optique d'une catégorisation linguistique, le seul terme dont on dispose actuellement est celui de parler bilingue. Le terme parler rend compte d'un assemblage particulier de variantes non standards et standards, tandis que celui de bilingue suppose la présence de deux systèmes linguistiques. C'est ainsi qu'à elle seule, la notion de parler bilingue peut renvoyer : 
- à la présence d'un système qui comporte la trace manifeste d'une langue différente de la langue de socialisation de la personne, et qui agit à des degrés divers sur le comportement langagier,

- au caractère variable des formes réalisées en LA,

— et enfin, à la compétence à jouer de l'ensemble du répertoire verbal.

Par ce choix conceptuel, on parvient à rendre compte de la langue des sujets tout en spécifiant le caractère bilingue du comportement et de l'usage qu'ils font de leur répertoire pluriel. Si l'on se fonde sur les théories sociolinguistiques dont les domaines de réflexion portent sur la description du parler bilingue en termes de marques transcodiques et sur les choix de langues, on retiendra le qualificatif de parler bilingue stabilise ${ }^{10}$. En effet, s'il est pour le moins étonnant de qualifier de système approximatif les connaissances linguistiques en LA des migrants (en référence à la langue cible), il est certainement plus juste d'y voir un système qui s'est stabilisé au terme d'un long processus d'acquisition. On rejoint ici la définition du bilingue telle qu'elle est envisagée par Py (1991 : 147), comme un locuteur achevé dont la compétence serait aussi stabilisée que celle d'un locuteur natif. Il est peut être vrai qu'une telle approche présente un côté abstrait et artificiel mais « ce n'est toutefois pas trop grave étant donné la relative lenteur avec laquelle évolue la langue » (Py 1980 : 74).

galliganistef@aol.com

\section{BIBLIOGRAPHIE}

ADJEMIAN, C. (1976) : «On the nature of interlanguage system », Language Learning 26 (2), 297-320.

BAUTIER-CASTAING, E. (1980) : «La notion de stratégie d'apprentissage permet-elle de rendre compte de l'acquisition d'une langue seconde par des enfants? », Langages 57, 95-105.

BERTHOUD, A-C., PY, B. (1979) : «Etude des processus d'apprentissage de L2. Problèmes méthodologiques et illustrations : contraintes déictiques sur l'emploi de quelques verbes de mouvement en français, allemand et espagnol », Encrages, numéro spécial Linguistique appliquée, 22-31.

BESSE, H., PORQUIER, R. (1991) : Grammaire et didactique des langues. Paris, Hatier, coll. LAL.

CORDER, S.P. (1980) : «La sollicitation de données d’interlangue », Langages 57, 29-37.

COSTA GALLIGANI, S. (1998 a) : Le français parlé par des migrants espagnols de longue date: biographies et pratiques langagières. Thèse de doctorat, Grenoble, Université Stendhal.

COSTA GALLIGANI, S. (1998 b) : «Comment j’ai appris le français: étude des représentations de migrants espagnols», dans J. BILLIEZ (dir.) : De la didactique des

${ }^{10}$ La stabilisation est donc à distinguer de la fossilisation (voir Costa Galligani 2001). 
Stéphanie Galligani

langues à la didactique du plurilinguisme, Hommage à Louise Dabène, Grenoble, CDLLIDILEM, 187-197.

COSTA GALLIGANI, S. (2001): «Compétences, productions et représentations métalinguistiques chez des migrants espagnols de longue date », Langue Française 131, 52-65.

FRAUENFELDER, U., NOYAU, C., PERDUE, C., PORQUIER, R. (1980) : «Connaissances en langue étrangère », Langages 57, 43-59.

FRAUENFELDER, U., PORQUIER, R. (1980) : «Le problème des tâches dans l'étude de la langue de l'apprenant », Langages 57, 61-71.

NEMSER, W. (1971) : "Approximative systems of foreign language learners », International Revue of Applied Linguistics IX-2,115-123.

NEUFELD, G.G. (1979) : "Vers une théorie de la capacité d'apprentissage linguistique », Encrages, numéro spécial Linguistique appliquée, 9-15.

NOYAU, C. (1976) : «Les 'français approchés' des travailleurs migrants : un nouveau champ de recherche », Langue Française 29, 45-60.

PORQUIER, R. (1977): L'analyse des erreurs : problèmes et perspectives », Etudes de Linguistique Appliquée 25, 23-43.

PORQUIER, R. (1994) : «Communication exolingue et contextes d'appropriation: le continuum acquisition/apprentissage », Bulletin Suisse de Linguistique Appliquée 59, 159169.

PY, B. (1980) : "Quelques réflexions sur la notion d'interlangue », Travaux Neuchâtelois de Linguistique 1, 31-55.

PY, B. (1991) : «Bilinguisme, exolinguisme et acquisition : rôle de L1 dans l'acquisition de L2 », Travaux Neuchâtelois de Linguistique 17, 147-161.

RATTUNDE, E. (1980) : «Stabilité vs instabilité des connaissances intermédiaires », dans Encrages. Acquisition d'une langue étrangère, Paris, Université Paris VIII - Vincennes, 46-53.

ROSEN, E. (1995) : Interprétation de la notion d'interlangue : analyse des représentations d'apprenants de français langue maternelle en formation professionnelle par alternance. Mémoire de D.E.A., Université Paris X - Nanterre.

SELINKER, L. (1972) : «Interlanguage », International Review of Applied Linguistics X-3, 209-231.

SELINKER, L., SWAIN, M., DUMAS, G. (1975) : «The interlanguage hypothesis extended to children », Language Learning 25-1, 139-152.

VOGEL, K. (1995) : L'interlangue : la langue de l'apprenant, Toulouse, Presses Universitaires du Mirail. 